\title{
Automated Facial Analysis
}

Joy Buolamwini

\section{Source}

Buolamwini, J. and Gebru, T. (2018). Gender Shades: Intersectional Accuracy Disparities in Commercial Gender

Classification. Proceedings of Machine Learning Research.

Automated facial image analysis describes a range of face perception tasks including, but not limited to, face detection, face classification, face recognition. 